Our Nature (2010) 8: 258-269

\title{
Physico-Chemical Studies on Chimdi Lake of Sunsari District during its Restoration Stage
}

\author{
R.Surana ${ }^{1}$, B.R Subba ${ }^{2}$ and K.P Limbu ${ }^{3}$ \\ Department of Zoology, P.G. Campus, T.U., Biratnagar, Nepal \\ E-mail: ${ }^{1}$ ranjanajain27@yahoo.com, ${ }^{2}$ subbabharat@yahoo.com, ${ }^{3}$ limbukp@gmail.com
}

Received: 15.06.2010, Accepted: 29.07.2010

\begin{abstract}
Chimdi Lake was ecologically a valuable site but due to the exploitation, almost all parts of the Lake changed into a terrestrial habitat. The issues of lake conservation have been brought into light recently and the work for rehabilitation; protection and conservation have been initiated by local peoples. Water quality plays an important role in determining the biodiversity of any water body. Various physico-chemical parameters such as atmospheric temperature, water temperature, transparency, total solids, total dissolved solids, total suspended solids, humidity, $\mathrm{pH}$, dissolved oxygen, free carbon dioxide, total alkalinity, acidity, total hardness, calcium hardness, calcium, magnesium and chloride were estimated on monthly basis from March 2004 to February 2005. Physico- chemical values revealed that the lake is a suitable site for the habitat of aquatic lives.
\end{abstract}

Key words: Physico-chemical, restoration, Chimdi Lake, Nepal

\section{Introduction}

Chimdi Lake is located in eastern Nepal. It is about $12 \mathrm{~km}$ west from Nepal's main industrial city Biratnagar. The lake lies between $87^{\circ} 10^{\prime} 51.3 " \mathrm{E}$ longitudes and $26^{\mathrm{O}} 29^{\prime} 23.5^{\prime \prime} \mathrm{N}$ latitude at an elevation of $70 \mathrm{~m}$ above the mean sea level. The total area of the lake is approximately 101.6 hectares.

The hydrological set up of any aquatic ecosystem plays a key role in determining the quality and productivity of any water body. Seasonal fluctuations of various physico-chemical factors have a crucial role in the distributions, periodicity, qualitative and quantitative composition of biota in aquatic ecosystem. The knowledge of all these factors is essential for identifying the suitability and fertility of an aquatic ecosystem.

The wetland serves as a habitat for a wide variety of waterfowls, fishes, wetland plants and vegetation. Chimdi Lake was also a rich habitat that supported rich biodiversity. In the absence of awareness on environmental issues, local people drained the water of this lake and converted a large part of it into paddy fields and wastelands.

Extensive works on different aspects of physico-chemical and biological parameters of fresh water body have been done in different parts of the world. Important works done outside of Nepal include the works of Ganpati (1943), Welch (1948), Mc. Combie (1953), Barret (1957), Spence (1964), Mc.Coll (1972), Jayaraman (1974), Swarup and Singh (1979), Weller 
R. Surana, B.R. Subba and K.P.Limbu / Our Nature (2010) 8: 258-269

(1981), Trivedy and Goel (1984), Khan and Chaudhary (1994) and Rawat et al. (1995).

Though vast number of limnological investigations has been carried out in different parts of the world, only few works have been done in Nepal. Some of the works are those of Loffler (1969), Ferro (1976), Bhandari (1992), Chhetri (1992), Shrestha (1992), Thapa (1994) and Udash (1996).

\section{Materials and Methods}

Preliminary survey of the study site was made. Two different sub-sites as station A and $\mathrm{B}$ were selected in the lake for the analysis of physico-chemical parameters of the lake water. Station A is located towards the eastern part of the lake and consists of low number of aquatic macrophytes. Station B is located towards the western part of the lake and has luxuriant growth of macrophytes. The study was carried out for a period of 12 months (March 2004 to February 2005). It was generally done between 9 to 11 am in the morning

The lake area, latitude, longitude and altitude of Chimdi Lake were measured by using e-trex Vista GPS.

For each station sample, temperature, transparency, dissolved oxygen $\left(\mathrm{DO}_{2}\right)$, free carbon dioxide $\left(\mathrm{FCO}_{2}\right)$, Total alkalinity (TA), Total Hardness (TH), Calcium Hardness $\left(\mathrm{Ca}^{++}\right)$, Acidity, Chloride, Euphotic limit, calcium and magnesium were all analyzed in the field.

Total solids, total dissolved solids and total suspended solids were determined in the laboratory within 24 hours. Physicochemical analysis of water was done as per standard methods recommended by Adoni (1985) and APHA (1998).
Statistical analysis was done by using computer software Genstat 5, Lawes Agricultural Trust (IACR- Rothamsted Experimental Station).

\section{Results}

Data on physico-chemical parameters have been tabulated in Table 1 and 2. Mean values along with \pm S. D. of physicochemical parameters have been presented in Table 3. Table 4 shows the coefficient of correlation values.

Physical Factors

(a) Temperature

Atmospheric temperature of the study area recorded were minimum $19 \pm$ $0.00^{\circ} \mathrm{C}$ (January) and maximum $34 \pm$ $0.00^{\circ} \mathrm{C}$ (September). The temperature started decreasing after September and reached minimum in January, after which it started increasing.

The water temperature fluctuated according to the atmospheric temperature which ranged from $18.8 \pm 0.00^{\circ} \mathrm{C}$ (January) to $30.5 \pm 0.42^{\circ} \mathrm{C}$ (September). No marked variations were observed between the sampling stations.

\section{(b) Humidity}

Related humidity ranged from $53 \pm 0.00 \%$ (December) to $88 \pm 0.00 \%$ (June).

\section{(c) Transparency}

Monthly variations in the Secchi disc transparency ranged between $2.13 \pm 0.00$ $\mathrm{cm}$ (July) and $27.83 \pm 0.00 \mathrm{~cm}$ (September), the minimum value in the monsoon and the maximum in the winter. 
(d) Euphotic Limit

The Euphotic limit value varied from $5.34 \pm 0.00 \mathrm{~cm}$ (July) to $69.58 \pm 0.00 \mathrm{~cm}$ (September).

\section{(e) Total Solids}

The concentration of total solid ranged from $200 \pm 0.00 \mathrm{mg} / \mathrm{L}$ (May) to 960 $\pm 56.56 \mathrm{mg} / \mathrm{L}$ (August), the minimum value in the summer and the maximum in the monsoon.

The concentration of total solid ranged from $200 \mathrm{mg} / \mathrm{L}$ (May) to $920 \mathrm{mg} / \mathrm{L}$ (August) in site $\mathrm{A}$. The value ranged from $200 \mathrm{mg} / \mathrm{L}$ (May) to $1000 \mathrm{mg} / \mathrm{L}$ (August) in site $\mathrm{B}$.

\section{(f) Total Dissolved solids.}

The concentration of total dissolved solids ranged from $100 \pm 0.00$

$\mathrm{mg} / \mathrm{L}$ (May) to $340 \pm 28.28 \mathrm{mg} / \mathrm{L}$ (August).

The maximum value recorded in site A and site B was $320 \mathrm{mg} / \mathrm{L}$ (August) and $360 \mathrm{mg} / \mathrm{L}$ (August) respectively.

(g) Total suspended solids.

The concentration of total suspended solids ranged from $100 \pm 0.00$ $\mathrm{mg} / \mathrm{L}$ (May) to $620 \pm 28.28 \mathrm{mg} / \mathrm{L}$ (August).

The maximum value recorded in site $\mathrm{A}$ and site $\mathrm{B}$ was $600 \mathrm{mg} / \mathrm{L}$ (August) and $640 \mathrm{mg} / \mathrm{L}$ (August) respectively.

\subsubsection{Chemical Factors}

(a) Hydrogen ion concentration $(\mathrm{pH})$ $\mathrm{pH}$ values of the lake water remained almost alkaline throughout the study period and varied from $6.05 \pm 0.028$ (April) to $8 \pm$ 0.70 (May). The variations in $\mathrm{pH}$ within sub sites were not considerable and ranged between 6.05 (April) to 8.5 (May).

(b) Dissolved Oxygen (DO2)

The dissolved oxygen contents showed its minimum value during summer (June) with its value of $4.82 \pm 0.56 \mathrm{mg} / \mathrm{L}$ and maximum during winter (September) with its value of $19.92 \pm 1.98 \mathrm{mg} / \mathrm{L}$.

The minimum value of $\mathrm{DO}_{2}$ in site $\mathrm{A}$ was $4.42 \mathrm{mg} / \mathrm{L}$ and site $\mathrm{B}$ was $5.22 \mathrm{mg} / \mathrm{L}$. The maximum value of $\mathrm{DO}_{2}$ in site $\mathrm{A}$ was $12.46 \mathrm{mg} / \mathrm{L}$ and in site $\mathrm{B}$ was $10.45 \mathrm{mg} / \mathrm{L}$.

(c) Free Carbon dioxide (FCO2)

The value of $\mathrm{FCO}_{2}$ ranged between $2.2 \pm 3.11 \mathrm{mg} / \mathrm{L}$ (January) to $30.8 \pm 0.00$ $\mathrm{mg} / \mathrm{L}$ (July).

Free carbon dioxide was found to be absent in the station B (January) and the lowest value recorded in station A was 4.4 $\mathrm{mg} / \mathrm{L}$ in the same month.

(d) Total Alkalinity

Total alkalinity was due to bicarbonate only. The value ranged between $32 \pm 0.00 \mathrm{mg} / \mathrm{L}$ (July) to $86 \pm 33.9 \mathrm{mg} / \mathrm{L}$ (March). Considerable difference within sub-sites was observed in some months.

(e) Total acidity

The value of total acidity ranged between $2.5 \pm 3.53 \mathrm{mg} / \mathrm{L}$ (January) to $35 \pm$ $0.00 \mathrm{mg} / \mathrm{L}$ (July).

(f) Total Hardness

The minimum value of total hardness recorded in the lake water was 38 $\pm 7.07 \mathrm{mg} / \mathrm{L}$ (June) and the maximum value recorded in the lake water was $140 \pm 26.62$ $\mathrm{mg} / \mathrm{L}$ (March).

The maximum value recorded in the 
R. Surana, B.R. Subba and K.P.Limbu / Our Nature (2010) 8: 258-269

site A was $156 \mathrm{mg} / \mathrm{L}$ and minimum value recorded was $32 \mathrm{mg} / \mathrm{L}$. Similarly, the maximum value recorded in site B was 124 $\mathrm{mg} / \mathrm{Land}$ minimum value recorded was 44 $\mathrm{mg} / \mathrm{L}$.

\section{(g) Calcium Hardness}

The value of calcium hardness ranged from $23 \pm 2.83 \mathrm{mg} / \mathrm{L}$ (June, October) to $84 \pm 28.28 \mathrm{mg} / \mathrm{L}$ (March).

The value of calcium hardness in site A ranged from $18 \mathrm{mg} / \mathrm{L}$ (June) to 104 $\mathrm{mg} / \mathrm{L}$ (March). The value of calcium hardness in site $\mathrm{B}$ ranged from $22 \mathrm{mg} / \mathrm{L}$ (September, October) $64 \mathrm{mg} / \mathrm{L}$ (March)

(h) Calcium $(\mathrm{Ca}++)$

Calcium values obtained by calculation ranged between $9.21 \pm 0.34$ $\mathrm{mg} / \mathrm{L}$ (June) to $33.66 \pm 11.33 \mathrm{mg} / \mathrm{L}$ (March).

In both the sites the minimum value was obtained in summer and maximum in winter.

\section{(i) Magnesium ( $\mathrm{Mg}++)$}

Magnesium values obtained by calculation ranged between $3.65 \pm 0.93$ $\mathrm{mg} / \mathrm{L}$ (June) to $13.66 \pm 1.38 \mathrm{mg} / \mathrm{L}$ (March).

The value in site A ranged between $3.41 \mathrm{mg} / \mathrm{L}$ (June) to $12.68 \mathrm{mg} / \mathrm{L}$ (March). The value of magnesium in site $B$ ranged between $3.41 \mathrm{mg} / \mathrm{L}$ (August) to $14.64 \mathrm{mg} / \mathrm{L}$ (March)

(j) Chloride

The concentration of chloride ranged from $4.92 \pm 1.004 \mathrm{mg} / \mathrm{L}$ (November) to $15.62 \pm 4.01 \mathrm{mg} / \mathrm{L}$ (March).

The value of chloride in site $A$ ranged between $5.68 \mathrm{mg} / \mathrm{L}$ (November) to $12.78 \mathrm{mg} / \mathrm{L}$ (March).

The value of chloride in site $B$ ranged between $4.26 \mathrm{mg} / \mathrm{L}$ (November) to $18.46 \mathrm{mg} / \mathrm{L}$ (March).

\section{Discussion}

The meteorological factors such as ambient temperature sunshine, rainfall, humidity exert a considerable influence on the physico-chemical dynamics of water body. The physico-chemical characteristic of water largely determines the structure and composition of biotic community of an aquatic ecosystem. Reid (1961) has stated that the successful development and maintenance of a population of organisms depends upon harmonious ecological balance between environmental conditions and tolerance of the organisms to variations in one or more of these conditions.

In the present study a strong positive and significant correlation $(\mathrm{r}=+$ $0.94, \quad \mathrm{P}<0.05)$ was found between atmospheric temperature and water temperature. Similar observations were made by Kant and Anand (1978) and Rawat. et al. (1995) . Welch (1952) and Munawar (1970) have observed that shallower the water body more quickly it reacts to the change in the temperature.

Transparency of water was higher after rainy season and lower values were observed during rainy season. Mc.Combie (1953) recorded that the transparency shows a direct relationship with the suspended organisms and nonliving particles in the water. During winter the higher value of transparency may be related with the settling rate of the suspended particles, absence of rain, run off, flood water and gradual decrease in plankton growth due to low temperature. Similar trend was observed by Rawat. et al. (1995) in Deoria Tal.

The mean value of total solids was higher during rainy season due to rain, run 
R. Surana, B.R. Subba and K.P.Limbu / Our Nature (2010) 8:258-269

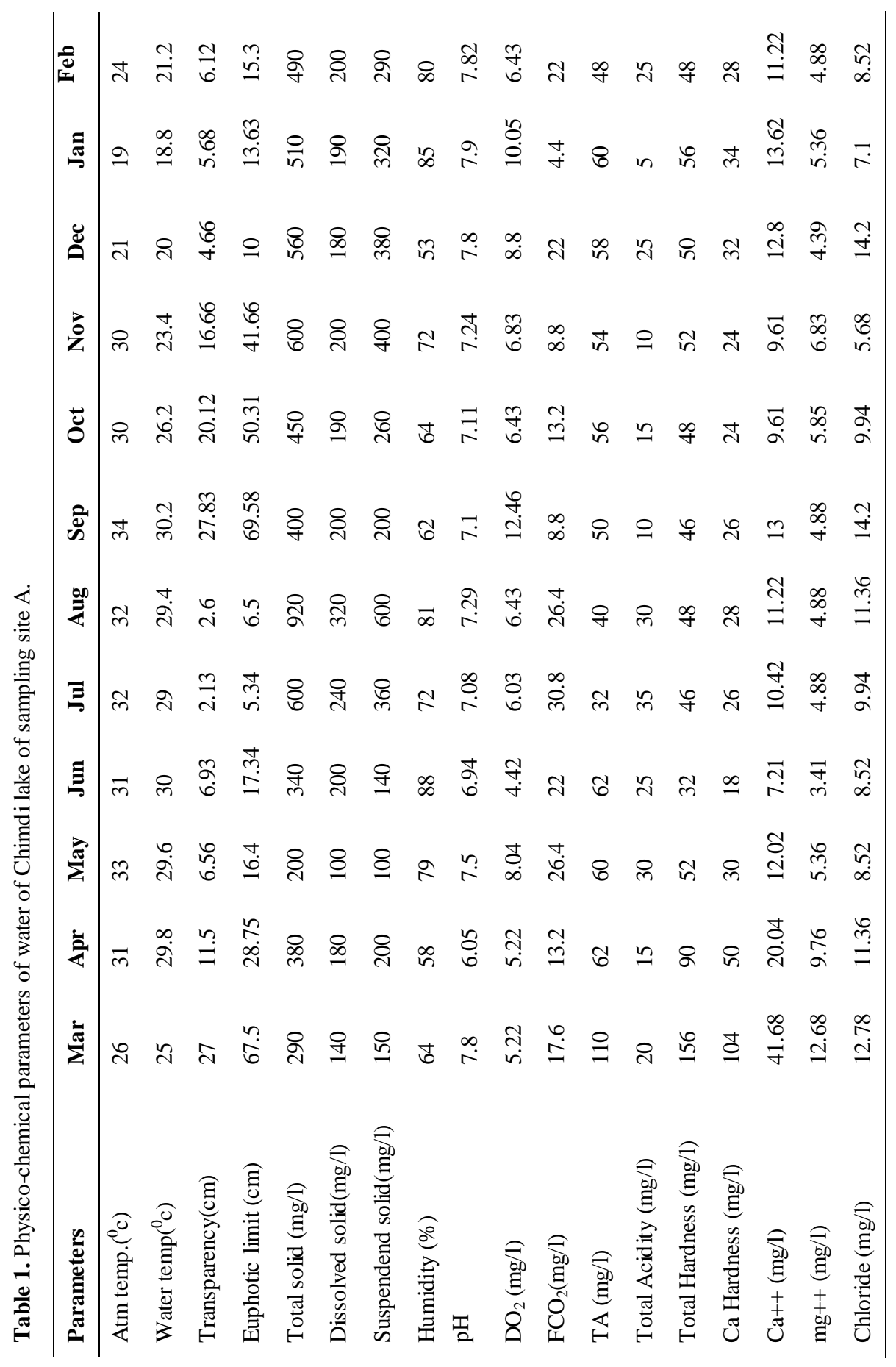


R. Surana, B.R. Subba and K.P.Limbu / Our Nature (2010) 8: 258-269

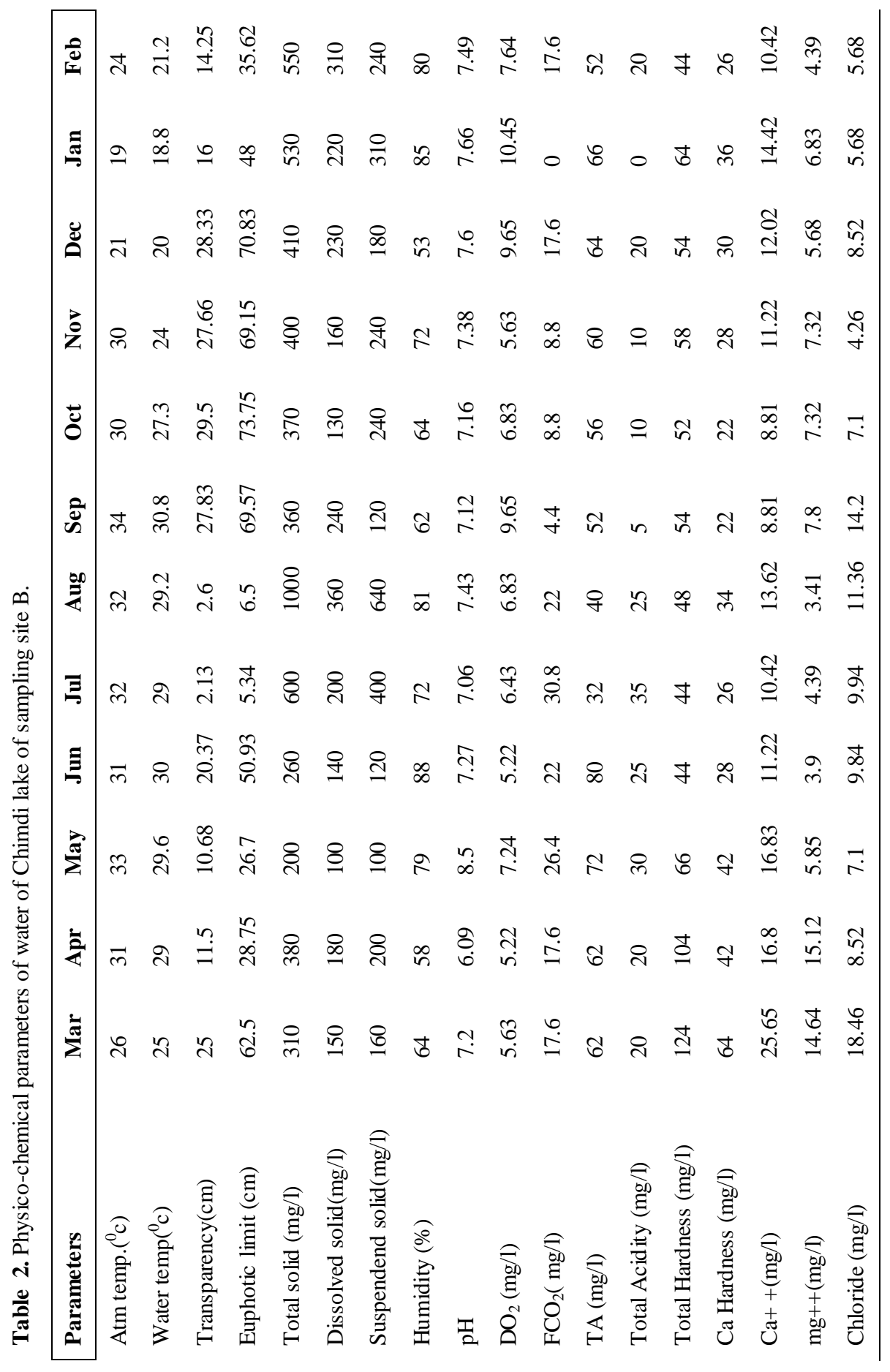


R. Surana, B.R. Subba and K.P.Limbu / Our Nature (2010) 8: 258-269

\begin{tabular}{|c|c|c|c|c|c|c|c|c|c|c|c|c|c|c|c|c|c|c|c|c|}
\hline & & & $\mid \begin{array}{c}0 \\
+1 \\
\stackrel{1}{*} \\
\stackrel{\lambda}{*}\end{array}$ & $\begin{array}{l}0 \\
+1\end{array}$ & $\begin{array}{l}+1 \\
t\end{array}$ & & $\int_{1}^{+1} \underset{1}{2}$ & 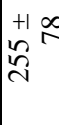 & 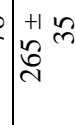 & $\begin{array}{l}0 \\
+1 \\
\infty \\
\infty\end{array}$ & $\begin{array}{l}0 \\
+1 \\
\stackrel{+}{+} \\
\end{array}$ & 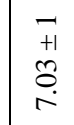 & $\begin{array}{l}m \\
+1 \\
\infty \\
\stackrel{0}{0}\end{array}$ & $\begin{array}{l}m \\
+1 \\
0 \\
n\end{array}$ & $\begin{array}{l}+ \\
+1 \\
n \\
i \\
\end{array}$ & $\begin{array}{l}m \\
+1 \\
o \\
o\end{array}$ & $\begin{array}{l}\overrightarrow{+} \\
\stackrel{+}{\sim}\end{array}$ & $\begin{array}{l}7 \\
+1 \\
\infty \\
0 \\
\varrho\end{array}$ & $\begin{array}{l}0 \\
+1 \\
\dot{+} \\
\dot{+}\end{array}$ & $\begin{array}{l}\stackrel{1}{+1} \\
\stackrel{+1}{\longrightarrow}\end{array}$ \\
\hline & & $\begin{array}{l}0 \\
+1 \\
2\end{array}$ & $\begin{array}{l}0 \\
+1 \\
\infty \\
\infty \\
\infty \\
\infty\end{array}$ & +1 & $\stackrel{+1}{\pi}$ & $\stackrel{m}{\dot{f}} \mid+\underset{\tilde{N}}{+}$ & 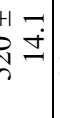 & 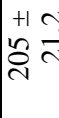 & 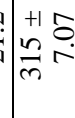 & $\infty$ & $\begin{array}{l}\overrightarrow{0} \\
\stackrel{+1}{r}\end{array}$ & 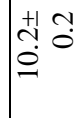 & $\begin{array}{l}+1 \\
\underset{c}{n} \\
\dot{c}\end{array}$ & 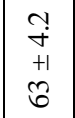 & 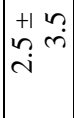 & $\begin{array}{l}0 \\
\dot{1} \\
+1 \\
\dot{8}\end{array}$ & $\begin{array}{l}+ \\
\stackrel{+}{+1} \\
\stackrel{m}{m}\end{array}$ & 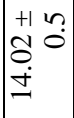 & 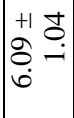 & 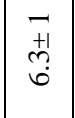 \\
\hline & & $\begin{array}{l}\stackrel{0}{0} \\
+1 \\
\dot{+}\end{array}$ & $\begin{array}{l}0 \\
\dot{0} \\
+1 \\
\stackrel{1}{2}\end{array}$ & \begin{tabular}{|l}
+1 \\
+1 \\
0 \\
0
\end{tabular} & $\dot{\dot{b}} \stackrel{+}{\stackrel{+}{\rightleftarrows}}$ & & $\begin{array}{lll}+1 & 0 \\
6 & 0 \\
0 & 8 \\
0 & 0\end{array}$ & 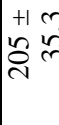 & 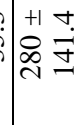 & $\begin{array}{l}8 \\
0 \\
+1 \\
n \\
n\end{array}$ & $\stackrel{+1}{\underset{丶}{\infty}}$ & $\begin{array}{c}0 \\
0 \\
+ \\
+1 \\
o \\
\sigma\end{array}$ & $\begin{array}{l}+1 \\
\infty \\
0 \\
2\end{array}$ & $\begin{array}{c}\mathcal{F} \\
+ \\
+1 \\
\sigma\end{array}$ & 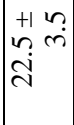 & $\begin{array}{l}\infty \\
i \\
+1 \\
+1 \\
i\end{array}$ & $\begin{array}{l}\stackrel{+}{+} \\
\frac{+1}{m}\end{array}$ & 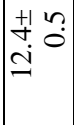 & 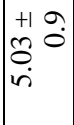 & 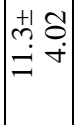 \\
\hline & & $\begin{array}{l}0 \\
0 \\
+1 \\
\dot{0}\end{array}$ & 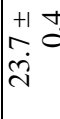 & $\mid \begin{array}{l} \pm 1 \\
\text { तi }\end{array}$ & $\begin{array}{l}+1 \\
\text { ti } \\
n\end{array}$ & 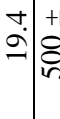 & 茎 & $\mid \begin{array}{ll}+1 & 0 \\
0 & \infty \\
0 & \ddots\end{array}$ & 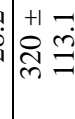 & $\begin{array}{l}\stackrel{0}{0} \\
+1 \\
i\end{array}$ & $\begin{array}{l}= \\
0 \\
+1 \\
\stackrel{+1}{r}\end{array}$ & \begin{tabular}{l}
$\infty$ \\
0 \\
+1 \\
\multirow{\jmath}{0}{}
\end{tabular} & $\left.\mid \begin{array}{ll}11 & 0 \\
\infty & 0 \\
\infty & 0\end{array}\right]$ & $\begin{array}{c}\sim \\
+ \\
+1 \\
i \\
i n\end{array}$ & $\begin{array}{l}\stackrel{0}{0} \\
+1 \\
\stackrel{0}{0}\end{array}$ & $\mid \begin{array}{ll}+1 & N \\
\text { in }\end{array}$ & $\begin{array}{l}\infty \\
i \\
+1 \\
+1 \\
\stackrel{N}{2}\end{array}$ & 告 & \begin{tabular}{ll}
+1 & 3 \\
$\vdots$ & 0 \\
\hdashline & 0
\end{tabular} & 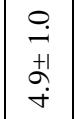 \\
\hline & & $\begin{array}{l}+1 \\
0\end{array}$ & 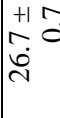 & $\mid \begin{array}{l}+1 \\
\infty \\
\infty \\
\dot{\sigma}\end{array}$ & $\begin{array}{l}1 \\
+ \\
6 \\
c \\
c\end{array}$ & 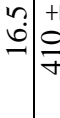 & 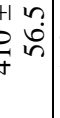 & {$\left[\begin{array}{ll}+1 & 7 \\
8 & 7 \\
- & 7\end{array}\right.$} & 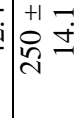 & $\begin{array}{l}0 \\
0 \\
+1 \\
0 \\
0\end{array}$ & 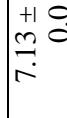 & 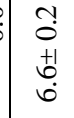 & $\begin{array}{l}\vec{m} \\
+1 \\
=\end{array}$ & $\mid \begin{array}{l}0 \\
0 \\
+1 \\
0 \\
0 \\
i\end{array}$ & $\begin{array}{lll}+1 & n \\
n & n \\
& m \\
& m\end{array}$ & $\begin{array}{l}\infty \\
\text { i } \\
+1 \\
+1 \\
i n\end{array}$ & $\begin{array}{l}\stackrel{+}{+} \\
\stackrel{+1}{+1} \\
\ddot{\vartheta}\end{array}$ & 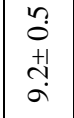 & 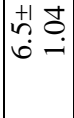 & 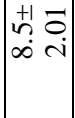 \\
\hline & & $\begin{array}{l}\stackrel{0}{0} \\
+1 \\
+ \\
\text { m }\end{array}$ & 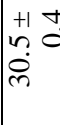 & 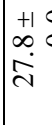 & $\mid \begin{array}{l}+1 \\
n \\
0 \\
0\end{array}$ & $\stackrel{\bullet}{\circ} \mid++$ & 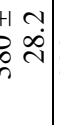 & 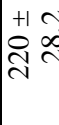 & $\begin{array}{lll}+1 & n \\
0 & 8 & 0 \\
0 & n\end{array}$ & $\begin{array}{l}8 \\
0 \\
+1 \\
\text { ปे }\end{array}$ & $\begin{array}{l}+1 \\
\stackrel{1}{\therefore} \\
\therefore\end{array}$ & $\mid$\begin{tabular}{ll}
+1 & 9 \\
\hdashline & -
\end{tabular} & {$\left[\begin{array}{l}+1 \\
\dot{0} \\
\dot{0}\end{array}\right.$} & $\begin{array}{c}+ \\
\dot{I} \\
+1 \\
i\end{array}$ & $\begin{array}{lll}+1 & n \\
r & n \\
r & n \\
r\end{array}$ & $\begin{array}{l}0 \\
i \\
+1 \\
+1 \\
i\end{array}$ & $\begin{array}{l}\stackrel{\infty}{i} \\
\text { + } \\
+1 \\
\stackrel{i}{+}\end{array}$ & $\begin{array}{l}+1 \\
\hat{a} \\
\dot{e}\end{array}$ & 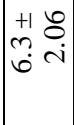 & $\begin{array}{ll}+1 & 0 \\
\text { I } & 0 \\
\pm & 0 \\
-\end{array}$ \\
\hline & & $\begin{array}{l}\stackrel{8}{0} \\
+1 \\
\text { cे }\end{array}$ & $\begin{array}{l}+1 \\
m \\
3 \\
m^{+1}\end{array}$ & $\mid \begin{array}{l}+1 \\
b \\
i \\
i\end{array}$ & ?ִ & & 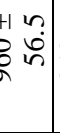 & $\begin{array}{ll}+1 & 0 \\
0 & \infty \\
m & \infty\end{array}$ & 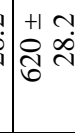 & $\begin{array}{l}8 \\
\dot{0} \\
+1 \\
\infty\end{array}$ & 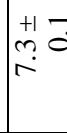 & $\mid \begin{array}{lll}+1 & 0 \\
0 & 0 & 0\end{array}$ & 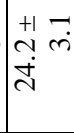 & $\mid \begin{array}{c}0 \\
0 \\
+1 \\
+ \\
q\end{array}$ & 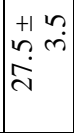 & $\begin{array}{c}8 \\
0 \\
+1 \\
\infty \\
o\end{array}$ & $\begin{array}{l}\stackrel{y}{+} \\
\stackrel{+}{+} \\
\stackrel{m}{+}\end{array}$ & $\begin{array}{l}+1 \\
+ \\
\text { İ } \\
\text { I }\end{array}$ & $\begin{array}{l}+1 \\
\stackrel{+}{+} \\
\dot{\sigma} \\
\dot{\theta}\end{array}$ & 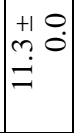 \\
\hline & & $\begin{array}{l}\dot{0} \\
+1 \\
\text { m }\end{array}$ & $\begin{array}{l}0 \\
\dot{0} \\
+1 \\
\text { iे }\end{array}$ & $\frac{+1}{i}$ & $\begin{array}{l}5 \\
9 \\
4 \\
4\end{array}$ & \begin{tabular}{l|l}
0 \\
$\dot{b}$ \\
-1 \\
\end{tabular} & $\begin{array}{lll}+1 & 0 \\
0 \\
0\end{array}$ & 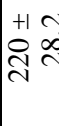 & 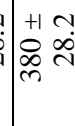 & $\mid \begin{array}{l}0 \\
0 \\
0 \\
+1 \\
i\end{array}$ & $\begin{array}{l}+1 \\
+1 \\
\vdots \\
\vdots \\
i\end{array}$ & $\mid \begin{array}{ll}+1 & 0 \\
& 0 \\
0\end{array}$ & 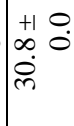 & $\begin{array}{l}0 \\
0 \\
+1 \\
i \\
\tilde{n}\end{array}$ & $\begin{array}{l}0 \\
0 \\
+1 \\
m \\
m\end{array}$ & 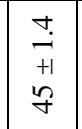 & $\begin{array}{l}0 \\
0 \\
+1 \\
+ \\
\stackrel{N}{1}\end{array}$ & $\begin{array}{l}+10 \\
+0 \\
0 \\
\stackrel{0}{0}\end{array}$ & $\mid \begin{array}{l}+1 \\
+1 \\
\dot{f} \\
\dot{f}\end{array}$ & \begin{tabular}{ll}
+1 & 0 \\
+ & 0 \\
\hdashline & 0
\end{tabular} \\
\hline & & $\begin{array}{l}8 \\
0 \\
+1 \\
\dot{m}\end{array}$ & $\begin{array}{l}8 \\
0 \\
+1 \\
0\end{array}$ & +1 & $\frac{+1}{m}$ & 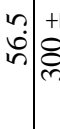 & 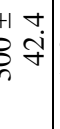 & $\mid \begin{array}{l}+1 \\
\stackrel{2}{2} \\
\underline{-}\end{array}$ & $\mid \begin{array}{ll}+1 & 0 \\
0 & 0\end{array}$ & $\begin{array}{l}8 \\
8 \\
+1 \\
\infty \\
\infty\end{array}$ & $+\underset{i}{+1} \approx$ & {$\left[\begin{array}{ll}+1 & n \\
\infty & 0 \\
\hdashline & 0\end{array}\right.$} & 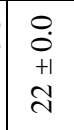 & $\begin{array}{l}0 \\
0 \\
+1 \\
i\end{array}$ & $\begin{array}{l}+ \\
\infty \\
+1 \\
+1 \\
d\end{array}$ & $\begin{array}{ll}+1 & 5 \\
\infty & 0 \\
m & -1\end{array}$ & $\begin{array}{l}\infty \\
i \\
+1 \\
\tilde{\lambda}\end{array}$ & $\begin{array}{lll}+1 & n \\
& m \\
\sigma & 0\end{array}$ & 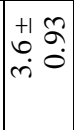 & $\begin{array}{ll}+1 & 0 \\
\text { I } & \stackrel{0}{0}\end{array}$ \\
\hline & & $\begin{array}{l}0 \\
0 \\
+1 \\
m\end{array}$ & 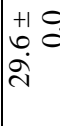 & $\mid \begin{array}{l}+1 \\
0 \\
\infty \\
\infty \\
\infty\end{array}$ & $\begin{array}{l}+1 \\
\text { in } \\
\text { in }\end{array}$ & 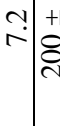 & 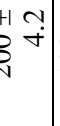 & $\begin{array}{ll}+1 & 0 \\
8 & 0\end{array}$ & $\mid \begin{array}{ll}+1 & 0 \\
8 & 0\end{array}$ & $\begin{array}{l}\stackrel{8}{0} \\
+1 \\
2 \\
2\end{array}$ & $\begin{array}{l}\hat{0} \\
\dot{0} \\
+1 \\
\infty\end{array}$ & $\begin{array}{l}n \\
0 \\
+1 \\
\stackrel{n}{0} \\
r\end{array}$ & $\begin{array}{ll}+1 & 0 \\
+ & 0 \\
\stackrel{0}{0} & 0\end{array}$ & $\mid \begin{array}{l}+ \\
\infty \\
+1 \\
+1 \\
0 \\
0\end{array}$ & $\begin{array}{l}0 \\
0 \\
+1 \\
0 \\
0\end{array}$ & $\begin{array}{l}2 \\
\hat{\sigma} \\
+1 \\
\hat{n}\end{array}$ & $\begin{array}{l}+ \\
\infty \\
+1 \\
+1 \\
b\end{array}$ & $\begin{array}{l}+1 \\
\stackrel{+}{+} \\
\dot{I}\end{array}$ & $\begin{array}{l}+10 ? \\
b \\
i n \\
\text { in }\end{array}$ & {$\left[\begin{array}{ll}+1 & 0 \\
\infty & 0 \\
i & -\end{array}\right.$} \\
\hline & & $\begin{array}{l}0 \\
0 \\
+1 \\
\dot{m}\end{array}$ & 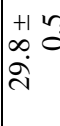 & $\begin{array}{l}+1 \\
n \\
\vdots \\
=\end{array}$ & 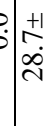 & & $\begin{array}{lll}+1 & 0 \\
0 & 0 & 0 \\
0\end{array}$ & $\begin{array}{lll}+1 & 0 \\
\infty & 0 \\
0 & 0\end{array}$ & $\mid \begin{array}{ll}+1 & 0 \\
\overbrace{i}^{2} & 0 \\
\end{array}$ & $\begin{array}{l}0 \\
0 \\
+1 \\
\infty \\
\infty\end{array}$ & 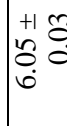 & 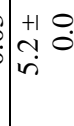 & $\begin{array}{l}+1 \\
+ \\
\ddot{n} \\
\ddot{n}\end{array}$ & $\begin{array}{c}0 \\
0 \\
+1 \\
\mathcal{O}\end{array}$ & $\begin{array}{ll}+1 & n \\
n & n \\
& m \\
& m\end{array}$ & $\begin{array}{l}2 \\
\hat{\alpha} \\
+1 \\
a\end{array}$ & $\begin{array}{l}0 \\
\dot{i} \\
+1 \\
+ \\
b\end{array}$ & $\begin{array}{l}-1 \\
0 \\
0 \\
0 \\
0 \\
0 \\
1\end{array}$ & 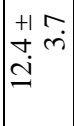 & $\begin{array}{ll}+1 & \overrightarrow{0} \\
\dot{t} & \vec{i}\end{array}$ \\
\hline & & $\begin{array}{l}0 \\
0 \\
+1 \\
+1 \\
0\end{array}$ & $\begin{array}{l}0 \\
0 \\
+1 \\
i \\
i\end{array}$ & $\begin{array}{l}+1 \\
+ \\
+1 \\
\text { D }\end{array}$ & 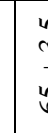 & $\begin{array}{l}? \\
\text { f } \\
6 \\
6\end{array}$ & 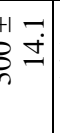 & $\begin{array}{l}+10 \\
110 \\
-9\end{array}$ & ن. & $\begin{array}{l}0 \\
\dot{0} \\
+1 \\
\text { I }\end{array}$ & 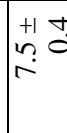 & $\begin{array}{l}+1 \\
+1 \\
y \\
i \\
i n\end{array}$ & $\begin{array}{ll}+1 & 0 \\
0 & 0 \\
\vdots & 0 \\
-\end{array}$ & $\begin{array}{ll}+1 & \sigma \\
\infty & \stackrel{m}{2}\end{array}$ & $\begin{array}{l}0 \\
0 \\
0 \\
+1 \\
0 \\
0\end{array}$ & $\begin{array}{ll}+1 & 0 \\
9 & i \\
- & \text { in }\end{array}$ & $\begin{array}{ll}+1 & \sim \\
+ & \infty \\
\infty & \sim\end{array}$ & $\begin{array}{l}+1 \\
\dot{+1} \\
\dot{m} \\
\dot{m}=\end{array}$ & 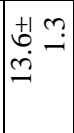 & 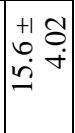 \\
\hline & & 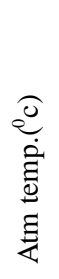 & 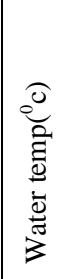 & 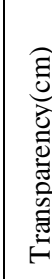 & 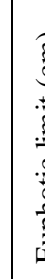 & 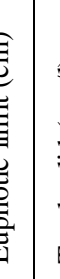 & 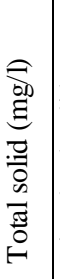 & 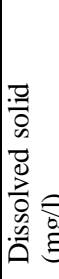 & 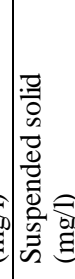 & & $\bar{a}$ & 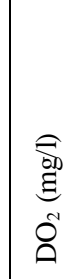 & 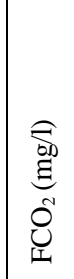 & 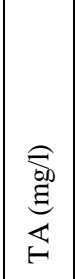 & 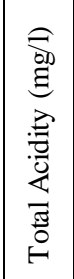 & 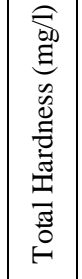 & 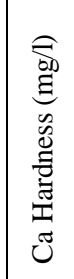 & 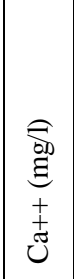 & $\begin{array}{l}\widehat{\text { bD }} \\
\Xi \\
\Xi \\
+ \\
+0 \\
\Xi\end{array}$ & 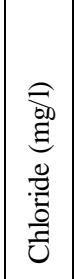 \\
\hline
\end{tabular}


R. Surana, B.R. Subba and K.P.Limbu / Our Nature (2010) 8: 258-269

\begin{tabular}{|c|c|c|c|c|c|c|c|c|c|c|c|c|c|}
\hline 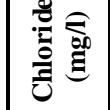 & $\stackrel{2}{\circ}$ & $\overline{\mathfrak{m}}$ & 导 & $\stackrel{5}{0}$ & $\stackrel{\infty}{0}$ & $\frac{7}{0}$ & $\overline{\check{o}}$ & $\frac{7}{0}$ & 京 & $\tilde{a}$ & in & $\hat{m}$ & , \\
\hline 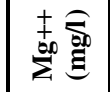 & $\stackrel{\overbrace{}}{\stackrel{i}{i}}$ & $\stackrel{8}{\circ}$ & $\bar{n}$ & 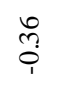 & त्रे & ત્̣ & 5 & ત્ર & t. & $\stackrel{\infty}{\infty}$ & 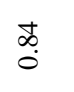 & & $\hat{m}$ \\
\hline 䓌哥 & $\stackrel{\vec{i}}{i}$ & 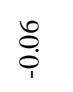 & \begin{tabular}{l}
$\infty$ \\
\multirow{1}{1}{}
\end{tabular} & $\stackrel{\Xi}{\circ}$ & 感 & $\overbrace{0}$ & : & $\ddot{\theta}_{0} r$ & s. & gे & & 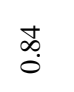 & $\stackrel{n}{a}$ \\
\hline 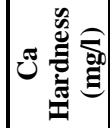 & $\begin{array}{l}0 \\
0 \\
i \\
i\end{array}$ & 㐫 & 㫄 & $\stackrel{0}{0}$ & त̂̀ & $\stackrel{\text { Oे }}{0}$ & : & ्ְ: & $\stackrel{0}{\circ}$ & ' & gे & $\stackrel{\infty}{\infty}$ & ֶี \\
\hline 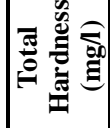 & $\underset{i}{+}$ & 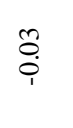 & के & $\stackrel{ \pm}{i}$ & స్ & $\stackrel{\circ}{i}$ & 㕝 & $\begin{array}{c}\circ \\
\vdots \\
i\end{array}$ & . & $\stackrel{0}{0}$ & ás & a & 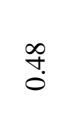 \\
\hline 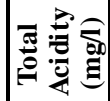 & $\widetilde{3}$ & 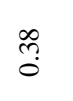 & $\stackrel{ \pm}{0}$ & ฮี & $\underset{\substack{\infty \\
+\\
i}}{0}$ & - & ָָ & ' & $\stackrel{9}{i}$ & $\stackrel{0}{0}$ & $\begin{array}{l}0 \\
\vdots \\
0 \\
0\end{array}$ & $\begin{array}{l}\text { ñ } \\
\text { ch } \\
\text { in }\end{array}$ & $\cong$ \\
\hline 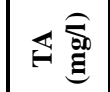 & तે & $\stackrel{7}{i}$ & $\stackrel{\infty}{\stackrel{0}{i}}$ & $\stackrel{\infty}{0}$ & $\stackrel{0}{i}$ & $\begin{array}{l}\text { an } \\
\text { chan }\end{array}$ & ' & $\begin{array}{l}n \\
\text { ñ. } \\
\text { on }\end{array}$ & $\begin{array}{l}t \\
0 \\
0\end{array}$ & 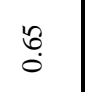 & $\stackrel{\mathscr{0}}{0}$ & in & $\overline{\text { ন্}}$ \\
\hline 总高 & త్రి & 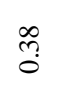 & $\stackrel{ \pm}{ \pm}$ & తి & 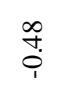 & & 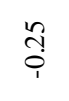 & - & $\stackrel{\circ}{i}$ & $\stackrel{8}{0}$ & 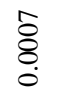 & 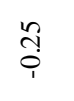 & $\cong$ \\
\hline 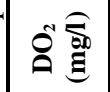 & $\begin{array}{c}\tilde{3} \\
\tilde{i}\end{array}$ & $\tilde{m}_{\hat{i}}$ & $\frac{9}{9}$ & $\stackrel{\circ}{0}$ & & $\stackrel{\infty}{\substack{+i}}$ & $\stackrel{0}{0}$ & f. & ণ্ডি & $\hat{\hat{S}}$ & ț & $\overrightarrow{\bar{i}}$ & $\stackrel{\infty}{0}$ \\
\hline $\mathrm{I}$ & 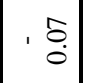 & $1 \frac{9}{0}$ & fof & , & స̂̀ & สุ & $\stackrel{\infty}{\circ}$ & $\delta$ & $\begin{array}{l} \pm \\
0 \\
0\end{array}$ & $\stackrel{8}{0}$ & $\bar{\partial}_{0}$ & ֻْ & $\stackrel{5}{\circ}$ \\
\hline 题 & ¿̊. & ठ̊. & & ?q & $\stackrel{2}{0}$ & $\stackrel{ \pm}{0}$ & $\stackrel{\infty}{0}$ & $\stackrel{ \pm}{0}$ & ले & : & ্ֻ] & $\vec{n}$ & $\stackrel{f}{0}$ \\
\hline 离产 & 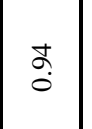 & & छे & $\stackrel{\circ}{\vec{i}}$ & 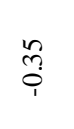 & $\stackrel{\infty}{0}$ & $\stackrel{\bar{c}}{\bar{i}}$ & ্ֻ & $\stackrel{\text { }}{\stackrel{\leftrightarrow}{i}}$ & $\stackrel{\infty}{\stackrel{\infty}{i}}$ & : & $\stackrel{\overbrace{}}{\circ}$ & $\bar{m}$ \\
\hline 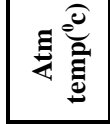 & ' & ga & : & : & $\tilde{\tilde{a}}$ & ల్రి & ڤ્તે & ల్లె & $\stackrel{ \pm}{i}$ & क्ञ̀ & $\stackrel{\infty}{\stackrel{0}{i}}$ & $\stackrel{\text { }}{\stackrel{i}{i}}$ & $\frac{n}{0}$ \\
\hline & 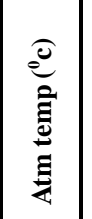 & 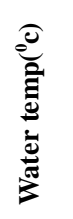 & 绨 & $\nexists$ & 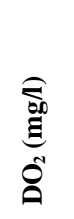 & 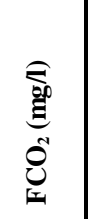 & $\bar{E}$ & 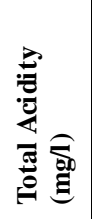 & 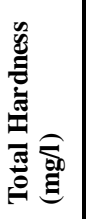 & 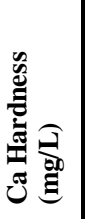 & 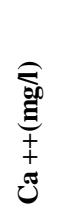 & 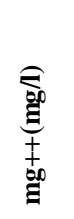 & 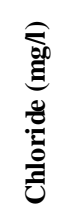 \\
\hline
\end{tabular}


R. Surana, B.R. Subba and K.P.Limbu / Our Nature (2010) 8: 258-269

off and sediment deposition. During summer minimum value was obtained. The same trend was obtained regarding total dissolved solid and total suspended solids. Rawat et al. (1995) has also computed maximum value of total dissolved solid in the month of August. The value of total dissolved solids $(100 \pm 0.00 \mathrm{mg} / \mathrm{L}$ to $340 \pm$ $28.28 \mathrm{mg} / \mathrm{L}$ ) was very less in comparison to the value obtained by Leghari et al. (2001) in Dhabeji springs, Malir, Karachi $(1943-2732 \mathrm{mg} / \mathrm{L})$.

In the present investigation $\mathrm{pH}$ showed insignificant inverse correlation with water temperature, total hardness and magnesium. Other physico-chemical parameters showed insignificant positive correlation with $\mathrm{pH}$.

Rawat et al. (1995) obtained positive significant correlation with total alkalinity $(\mathrm{r}=+0.52, \mathrm{P}<0.05)$ but such correlation was not obtained in the present study.

Bist (1993) opined that variation in $\mathrm{DO}_{2}$ could be due to one or more factors such as temperature, light intensity, turbidity, photosynthesis and respiration. The values of $\mathrm{DO}_{2}$ are higher in winter and lower in summer. However in present study higher value was obtained in the month of September. This may be due to luxuriant growth of macrophyte thus increase in photosynthesis rate than respiration.

The dissolved oxygen showed inverse but insignificant correlation with water temperature $(\mathrm{r}=-0.3)$, such observation was also shown by Jindal and Kumar (1993) and Thapa (1994). According to McColl (1972) the relation between temperature and dissolved oxygen is not so significant because the production and consumption of oxygen takes place simultaneously.

The value of $\mathrm{FCO}_{2}$ decreased from summer to rainy and then increased. The permitted level of $\mathrm{FCO}_{2}$ is 4 to $25 \mathrm{mg} / \mathrm{L}$.

The absence of $\mathrm{CO}_{2}$ during January on site B may be chiefly due to its complete consumption in carbon assumption or its complete conversion into carbonic acid and ultimately into stable carbonate and bicarbonate.

Maximum $\mathrm{FCO}_{2}$ was observed during monsoon may be accounted by the reduced photosynthesis during these months. The increase concentration of $\mathrm{FCO}_{2}$ at this time also indicates its influx through rain water in the form of carbonic acid, Chakraborty (1959) also supports his agreement.

In the present investigation free carbon dioxide shows inverse correlation ( $\mathrm{r}$ $=+0.4$ ) with $\mathrm{DO}_{2}$ and the same was observed by Ganpati (1943) and Pandey and Lal (1995). $\quad \mathrm{FCO}_{2}$ shows positive but insignificant correlations with water temperature $(\mathrm{r}=+0.3, \mathrm{P}<0.05)$ as by Rao (1955). However in the present investigation $\mathrm{FCO}_{2}$ does not show any distinct correlation with other physicochemical parameters.

In the present investigation the mean value of alkalinity obtained ranged between $32 \pm 0.00 \mathrm{mg} / \mathrm{L}$ to $86 \pm 33.9 \mathrm{mg} / \mathrm{L}$ which was lower than those reported by Pandey and Lal (1995). Total alkalinity showed positive and significant correlation with total hardness $(\mathrm{r}=0.6, \mathrm{P}<0.05)$, positive and significant correlation with calcium hardness $(\mathrm{r}=0.6, \mathrm{P}<0.05)$, positive and significant correlation with calcium $(r=0.65, P<0.05)$ and magnesium 
$(\mathrm{r}=0.5704, \mathrm{P}<0.05)$. Total alkalinity does not show any significant correlation with pH as observed by Rawat et al. (1995).

Spence (1964) working on the south Scottish lakes categorized lakes with more than 60ppm alkalinity as nutrient rich. Alkalinity in itself is not harmful to human beings; still the water suppliers with less than $100 \mathrm{mg} / \mathrm{L}$ are desirable for domestic use, Trivedy and Goel (1984). The present investigation shows that the lake water is suitable for aquatic life. In the present investigation acidity showed strong positive and significant correlation with free carbon dioxide $(\mathrm{r}=0.99, \mathrm{P}<0.05)$. Other physicochemical parameters showed no any significant correlation with acidity.

Higher value of total hardness was recorded in the month of March and lower value in the month of June. Dead Molluscan shell also releases chemical contents which increase the concentration of total hardness (Khan and Chowdhary,1994). In the present investigation total hardness showed positive and significant correlation with total alkalinity $(\mathrm{r}=0.64, \mathrm{P}<0.05)$, positive and significant correlation with calcium hardness $(r=0.96, P<0.05)$, positive and significant correlation with calcium $(\mathrm{r}=$ $0.97, \mathrm{P}<0.05)$ and positive and significant correlation with magnesium $(\mathrm{r}=0.94, \mathrm{P}<$ $0.05)$.

A water body having more than 25 $\mathrm{mg} / \mathrm{L}$ of calcium content has been regarded as rich by Ohle (1956). Calcium forms a principal component of shells of molluscs. The lake showed richness in calcium content thus suitable for molluscs which are good food for waterfowls.In the present investigation calcium hardness showed positive and significant correlation with total alkalinity $(\mathrm{r}=0.65, \mathrm{P}<0.05)$, positive and significant correlation with magnesium $(\mathrm{r}=0.83, \mathrm{P}<0.05)$, positive and significant correlation with chloride $(\mathrm{r}=0.52, \mathrm{P}<$ $0.05)$.

Magnesium occurs in all kinds of natural waters with calcium, and its concentration remains generally lower than calcium (Trivedy and Goel, 1984). The same trend was found in present investigation. The importance of magnesium lies with the chlorophyll bearing algae and plants. In the present investigation magnesium showed significant and positive correlation with total alkalinity $(\mathrm{r}=0.57, \mathrm{P}$ $<0.05)$ and positive and significant correlation with total hardness $(\mathrm{r}=0.94, \mathrm{P}<$ $0.05)$.

Chlorides are usually present in low concentration in water and play metabolically active role in photolysis of water and phosphorylation reactions in autotrophs. Higher chloride values were recorded during summer however no definite pattern of fluctuations, Swarup and Singh (1976) also reported an increase in Chloride during summer. The chloride concentration of lake water depends upon the amount of pollution causing matter entering into the lakes. The value obtained in the present study indicates that the lake is not polluted and lies within the tolerance limit $(28 \mathrm{mg} / \mathrm{L})$. In the present investigation chloride showed positive correlation with water temperature $(\mathrm{r}=0.32)$ but the relation is not significant. Chloride showed positive and significant correlation with calcium hardness $(\mathrm{r}=0.52, \mathrm{P}<0.05)$.

\section{References}

Adoni, A.D.C. (1985). Workbook on limnology, 
R. Surana, B.R. Subba and K.P.Limbu / Our Nature (2010) 8: 258-269

Bandana Printing service, New Delhi.

APHA (1998). Standard Methods for the examination of water and waste water, $20^{\text {th }}$ Edition, APHA, AWWA, WEF, Washigton DC.

Barrett, P.H. (1957). Potassium Concetration in fertilized trout lakes, limol. Oceanogr, 26:55 59.

Bhandari, B. (1992). The current status of wetland in Nepal. Country report presented at Asian wetland symposium, organized by Ramsar Center, Otsukushiro.

Bist, K.L. (1993). Environmental Parameters and Seasonal Succession in Planktonic Biomass in the River Finder of Garhawal Himalaya. In $\mathrm{H}$. R. Singh, (Ed.) In Advance in Limnology, 163 - 170, Narendra Publishing House, Delhi.

Chakraborty, R., D. P. Ray and S. B. Singh (1959). A Quantitative Study of the Plankton and Physicochemical Conditions of the River Jamuna at Allahabad in 1954 - 55. Ind. $J$. Fish 6 (1):186 - 203.

Chhetri, K. (1992). Study on the abundance and distribution of zooplankton of a village pond (Bhimpokhari), Kritipur, Department of zoology, T.U. Kathamdnu, Nepal. (M.Sc. Dissertation)

Ferro, W. (1976). Some limnological and biological data from Rara, a deep Himalayan lake in Nepal. Journal of the Nepal Research Resource Center 2 (3): 241 - 261, Tribhuvan University, Kathmandu, Nepal.

Ganpati, S. V. (1943). An ecological study of a garden pond containing abundant zooplankton, proc. Ind. Acad. Sci. 17:41 - 58.

Jayaraman, K.C. (1974). Ecology and distribution of freshwater fishes, Amphibian and Reptiles, In Ecology and Biogeogrophy in India. pp.517 884.

Jindal, R and R. Kumar (1993). Limnology of a freshwater pond of Nalagarh (Himachal Pradesh India). 1. Physico-Chemical Complexes. In H. R. Singh (Ed.) In Advances in Limnolgy, pp.107 - 112. Narendra
Publishing House, Delhi.

Kant, S and V.K., Anand (1978). Interrelationship of Phytoplankton and Physical Factors in Ansar Lake, Janmu (Jandk). Indian J. Ecology 5 (2):134 - 140 .

Khan, M.A.G. and S. H. Chowdhary (1994). Physical and chemical limnology of lake Kaptai Bangladesh, Tropical Ecology 35 (1): 35 - 51.

Leghari, S.M., T.M. Jahangir, M.Y. Khahawar and A. Leghari (2001). Physico-chemical and Biological study of Dhabeji spring, Malair, Karachi, Sindh., Pakistan, On Line Journal of Biological Sciences, 1 (7) : 623 - 627.

Loffler, H. (1969). High altitude lakes in the Mt. Everest Region, Verh international Verein limnolgoy $17: 373-385$.

Mc Combie, A. M. (1953). Factors Influencing the growth of Phytoplankton. J. Fish, Res. Bol. Cand. $10: 253-282$.

Mc.Coll, R.H.S. (1972). Chemistry and Trophic satus of Seven New Zealand Lakes. N. Z. J. Mar. Fresh W. Res. $6: 399$ - 447.

Munawar, M. (1970). Limnological studies in Freshwater ponds of Hyderabad, India, The Biotope, Hydrobiol. 35 : 127 - 162.

Ohle, W. (1956). Bioactivity production and energy utilization of lakes. J. Limnol Oceanog. 1 : $139-149$.

Pandey, K. K. and M.S. Lal (1995). Limnological studies of Garhwal Himalayan Hill Stream Khanda Gad : Seasonal Fluctuation in Abiotic Profile. J. Fresh Water Biol 7 (1): 7 - 11.

Rao, C. B. (1955). On the distribution of Algae in a group of six small ponds. Indian J. Ecol. 41 : $62-71$.

Rawat, M.S., C.P. Juyal and R. C. Sharma (1995). Morphometry and Physico-Chemical Profile of High Altitude Lake Deoria Tal of Garhwal Himalaya, J. Fresh Water Biol. 7 (1) : 1 - 6.

Reid, G. K. (1961). Ecology in Inland Waters and 
R. Surana, B.R. Subba and K.P.Limbu / Our Nature (2010) 8: 258-269

Estuaries, New York.

Shrestha, T.K. (1992). Conservation status of Swamp Patridge (Francolinus gularis) in Nepal. Gibier Faune Sauvage, 9: 553 - 560.

Spence, D.H.N. (1964). The Macrophytes Vegetation of freshwater swamps of scotland. In J.R. Bruneu (Ed.) Oliver and Boyd, pp.425,Edinburgh and London.

Swarup, K and Singh, S.R. (1976). Limnological Studies on Suraha Lake (Ballia). J. Inland Fish Soc. India 11(1): 22 - 23.

Thapa, T.B. (1994). An ecological study of village pond of Kirtipur with reference to water quality and zooplankton, Central Department of Zoology, Tribhuvan University, Kirtipur,
Kathmandu (M.Sc. Dissertation)

Trivedi, R. K. and P. K. Goel (1984). Chemical and Biological Methods for Water Pollution Studies, Environmental Publications, Karad, India.

Udash, R.K. (1996). Studies on ecological Characteristics of Tamor lake in Royal Chitwan National Park, Central Department of Zoology, Tribhuvan University, Kirtipur, Kathmandu (M.Sc. Dissertation)

Welch, P.S. (1952). Limnological Methods, Mc. Graw Hill Book, Co., New York.

Weller, M.W. (1981). Fresh Water Marshes Ecology and Wildlife Management, University of Minnesota Press, Minneapolis. 